\title{
Unravelling the role of amino acid sequence order in the assembly and function of the amyloid- $\beta$ core
}

Article

Accepted Version

Bera, S., Arad, E., Schnaider, L., Shaham-Niv, S., Castelletto, V., Peretz, Y., Zaguri, D., Jelinek, R., Gazit, E. and Hamley, I. W. (2019) Unravelling the role of amino acid sequence order in the assembly and function of the amyloid- $\beta$ core. Chemical Communications, 55 (59). pp. 8595-8598. ISSN 1359-7345 doi: https://doi.org/10.1039/C9CC03654G Available at https://centaur.reading.ac.uk/85148/

It is advisable to refer to the publisher's version if you intend to cite from the work. See Guidance on citing.

To link to this article DOI: http://dx.doi.org/10.1039/C9CC03654G

Publisher: Royal Society of Chemistry

All outputs in CentAUR are protected by Intellectual Property Rights law, including copyright law. Copyright and IPR is retained by the creators or other copyright holders. Terms and conditions for use of this material are defined in the End User Agreement. 


\section{CentAUR}

Central Archive at the University of Reading

Reading's research outputs online 


\section{Unravelling the role of amino acid sequence order in the assembly and function of the amyloid- $\beta$ core $\uparrow$}

Received 00th January 20xx, Accepted 00th January 20xx DOI: $10.1039 / \times 0 \times x 00000 x$
Santu Bera, ${ }^{\mathrm{a}}$ Elad Arad, ${ }^{\mathrm{b}}$ Lee Schnaider, ${ }^{\mathrm{a}}$ Shira Shaham-Niv, ${ }^{\mathrm{a}}$ Valeria Castelletto, ${ }^{\mathrm{c}}$ Yossef Peretz, ${ }^{\mathrm{b}}$ Dor Zaguri, ${ }^{a}$ Raz Jelinek, ${ }^{\text {b }}$ Ehud Gazit, ${ }^{* a}$ and Ian W. Hamley*c
The amino acid sequence plays an essential role in amyloid formation. Here, using the the central core recognition module of the $A \beta$ peptide and its reverse sequence, we show that although both peptides assemble into $\beta$-sheets, their morphologies, kinetics and cell toxicities display marked differences. In addition, the native peptide, but not the reverse one, shows notable affinity towards bilayer lipid model membranes that modulates the aggregation pathways to stabilize the oligomeric intermediate states and function as the toxic agent responsible for neuronal dysfunction.

There is an intense effort to elucidate the mechanism formation of amyloid structures by proteins and peptides, motivated by its relevance to understanding the misfolded structures, believed to cause diseases including Alzheimer's. ${ }^{1}$ The formation of fibrillar and oligomeric states of the Amyloid $\beta(\mathrm{A} \beta)$ peptide is implicated in the progression of this condition. The substantial research activity on $\mathrm{A} \beta$ has led to identification of a core aggregation domain which encompasses the $A \beta(16-22)$ AcKLVFFAE- $\mathrm{NH}_{2}$ sequence. ${ }^{1,2}$ This peptide has been shown to form fibrils or nanotubes, depending on the solution conditions. ${ }^{3}$ The core domain includes the FF sequence, which is believed to drive aggregation due to aromatic stacking interactions. ${ }^{4}$ The analogous peptide $\mathrm{NH}_{2}$-AAKLVFF-OH was also found to form fibrils and nanotubes in different conditions. ${ }^{5}$

The oligomerization propensity of $A \beta$-driven peptides is known to be related to their interaction with lipid bilayers. ${ }^{1 \mathrm{~d}, 6}$ Lipid membranes may (partially) unfold the peptide, increase the local concentration of membrane-bound peptide molecules, and facilitate aggregation by aligning the peptide. In addition, lipid

\footnotetext{
a. Department of Molecular Microbiology and Biotechnology, George S. Wise Faculty of Life Sciences, Tel Aviv University, Tel Aviv 69978, Israel; E-mail: ehudg@post.tau.ac.il

b. Department of Chemistry \&, Ilse Katz Institute (IKI) for Nanoscale Science and Technology, Ben Gurion University of the Negev, Beer Sheva 8410501, Israel

c. Department of Chemistry, University of Reading, Reading RG6 6AD, United Kingdom.E-mail; I.W.Hamley@reading.ac.uk

+ Electronic Supplementary Information (ESI) available: Experimental details, CD, SAXS, HPLC, MASS. ESI S1-7, Table S1. See DOI: 10.1039/x0xx00000x
}

rafts are implicated in $A \beta$ dimer and oligomer formation and may provide platforms for selective deposition of different $A \beta$ aggregates.

In the field of amyloid assembly, the influence of the sequence order has rarely been systematically examined. ${ }^{7}$ Reverse full A $\beta$ peptides $A \beta(42-1)$ and $A \beta(40-1)$ are sometimes used as negative controls in AD-related animal cytotoxicity studies and in $\mathrm{A} \beta$ production assays. ${ }^{7}$ Similarly, the reverse $A \beta(35-25)$ peptide is used as a control in studies using $A \beta(25-35){ }^{7,8}$ The retro-inverse $\mathrm{A} \beta$ peptide ffvlk (D-amino acids) has been shown to bind $A \beta 40$ fibrils with moderate affinity, but this binding can be significantly enhanced by attaching multiple copies of this peptide to an eight-arm branched PEG. ${ }^{9}$ In a recent study on a different amyloid peptide, the aggregation of a transthyretin fragment conjugated to RGD was compared to that of the reverse peptide, and both were found to form similar fibrillar assemblies. ${ }^{10}$ Serpell and coworkers have recently studied the self-assembly of the full length $\mathrm{A} \beta 1-42$ and its reverse sequence. ${ }^{7 a}$ Although both the peptides found to self-assemble into fiber structures, the reverse sequence has reduced effect on cellular health.

Here, for the first time, we compare the conformation and aggregation of $A \beta(16-22)$, the core recognition motif of $A \beta$ and the reverse peptide $A \beta(22-16)$. In addition, the cytotoxicity of the two peptides towards model neuronal cell line is investigated. Significant differences in the self-assembly kinetics and neural toxicity are observed. We further study the interactions of both pseudo-zwitterionic peptides with a model zwitterionic lipid mixture. The native peptide shows strong affinity towards lipid vesicles, resulting in stabilization of the oligomeric state by modulation of the aggregation kinetics thereby leading to toxicity. However, the reverse peptide has minimal affinity for the bilayer lipid vesicles as it self-assembles very quickly into mature fibers, which in turn are non-toxic to cells.

We first investigated the morphology of self-assembled nanostructures fabricated by the two peptides at neutral $\mathrm{pH}(\sim 7)$ after aging for two weeks. Fig. 1a-f shows transmission electron micrograph (TEM) and atomic force micrograph (AFM) images 
of the peptides. Amyloid-like straight, unbranched fibers 10-15 $\mathrm{nm}$ in diameter and length reaching to several microns were observed for both peptides. The CD spectra of both peptides showed typical peaks of a $\beta$-sheet secondary structure. The spectrum for the native peptide contained negative maxima at $217 \mathrm{~nm}$ with a positive peak at $202 \mathrm{~nm}$ (Fig. 1g). For the reverse peptide, the negative maxima was observed at $218 \mathrm{~nm}$, with a positive peak at $198 \mathrm{~nm}$ (Fig. 1h). Thus, both the peptides selfassembled to produce a $\beta$-sheet structure, with the peaks slightly red shifted as compared to canonical $\beta$-sheet probably due to strong $\pi-\pi$ stacking interactions. The high conformational flexibility of the peptide backbone was evaluated by temperature dependent denaturation experiments (ESI Figure S1, S2). Fourier transform infrared (FTIR) spectroscopy was employed to further confirm the preferred secondary structure. The native peptide showed a vibrational band at $1627 \mathrm{~cm}^{-1}$ in the amide I region, in correlation with a $\beta$-sheet conformation (Fig. 1i). The reverse peptide showed a peak at $1628 \mathrm{~cm}^{-1}$ with a shoulder at $1664 \mathrm{~cm}$ ${ }^{1}$, also indicating the predominate presence of a $\beta$-sheet structure (Fig. 1j).

To evaluate the details of the peptide molecular packing in the self-assembled fibers, small angle X-ray scattering (SAXS) was carried out. The SAXS intensity profiles (ESI Figure S3) showed an initial $\mathrm{q}^{-2}$ slope for both samples, consistent with flattened nanotape-like fibrils. Correspondingly, the data was fitted to a suitable form factor used for peptide nanotape fibrils. ${ }^{11}$ The results showed a clear differences between the molecular arrangement of the two peptides. The polydispersity in fibril diameter was much higher for $A \beta(16-22)$ in comparison to $\mathrm{A} \beta(22-16)$, and for the later a form factor maximum allowed an estimation of nanotape thickness, $(42.0 \pm 9.7) \AA$, corresponding to approximately one bilayer based on the expected length of a $\beta$-sheet heptapeptide (7x $3.2 \AA=22 \AA$ ). The fibril dimensions are in agreement with the TEM and AFM imaging (Fig. 1).

The kinetics of fiber formation was monitored by TEM imaging. ${ }^{12}$ Starting from small nucleation sites at $4 \mathrm{~h}$, the native peptide transformed into mature fibers through intermediate aggregation steps over a time period of four to seven days (Fig. 2a). In contrast, the reverse sequence showed structure formation at a much faster rate (Fig. 2b). Ordered structures were observed after $17 \mathrm{~h}$, and the self-assembly process was complete within four days. Thus, the intermediate aggregation rate of oligomers was significantly faster for the reverse peptide. The assembly process was further studied by following the CD spectra over time. Initially, the native peptide showed a negative peak near $200 \mathrm{~nm}$, resembling a random coil conformation. Time-induced structural conversion from random coil into $\beta$-sheet ellipticity was identified after 4 days (Fig. 2c). The molar ellipticity became stronger over time and plateaus after 10 days. The timedependent conformational transition from random coil to $\beta$-sheet is similar to that of other previously studied $A \beta$-derived peptides. ${ }^{13}$ The reverse peptide also elicited a similar structural transition, but with a different kinetics. Under similar conditions, the reverse peptide transformed from random coil into characteristic $\beta$-sheet ellipticity in 1 day (Fig. 2d). Over time, the peaks red shifted due to either strong aggregation through $\pi-\pi$ stacking interaction of the aromatic residue or twisting of the $\beta$ -
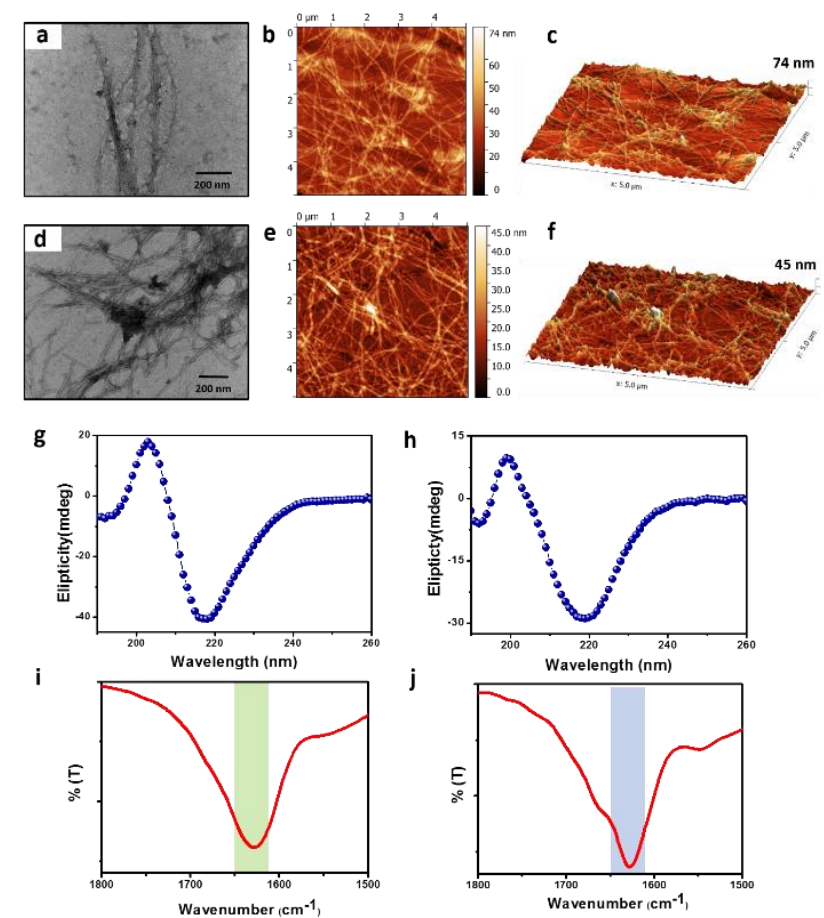

Fig. 1 (a) TEM and (b,c) AFM images of $A \beta(16-22)$. (d) TEM and (e,f) AFM images of $A \beta(22-16)$. (g,h) CD spectra for $A \beta(16-22)(g)$ and $A \beta(22-16)(h)$. (i, j) FTIR spectra for the native peptide (i) and its reverse sequence $(\mathrm{j})$.

sheet conformation during fiber formation. ${ }^{\text {b }}$ The kinetics of $\beta$ sheet formation by the two peptides, as shown by $\mathrm{CD}$, was consistent with the morphological transition observed in the TEM images. However, it is extremely difficult to explain the different aggregation rates of the two peptides, as the molecular events involved in the aggregation process of oligomers still remain poorly defined. Also, the higher negative $\zeta$-potential values for the reverse peptide, compared to the native peptide (Fig. 3a), indicated different surface charges and electrostatic repulsion between the molecules for the two peptides in the selfassembled state.

To explore whether these fibrillation processes play a role in the cytotoxicity of the corresponding peptides, similar to amyloid aggregates, we performed a 3-(4,5-dimethylthiazolyl-2)-2,5diphenyltetrazolium bromide (MTT) cell viability assay using the SH-SY5Y neuronal cell line. In the presence of the native peptide, cell viability decreased to approximately $70 \%$ within a short period of time $(6 \mathrm{~h})$, indicating substantial cell cytotoxicity (Fig. 3b). However, under similar experimental conditions, the reverse peptide showed no effect on the cells akin to the control experiment. A similar toxicity profile for the two peptides was also observed for another cell line, namely PC12 (ESI Figure S4). The differences in the toxicity are probably due to the intermediate oligomers formed by the native peptide during the fibrillization pathway, leading to toxicity. The mechanism of amyloidogenic protein/peptide aggregation typically involves a conformational switch, with oligomer formation leading to protofibrils and finally mature fibrils. Although the mature fibers were initially believed to be responsible for disease 

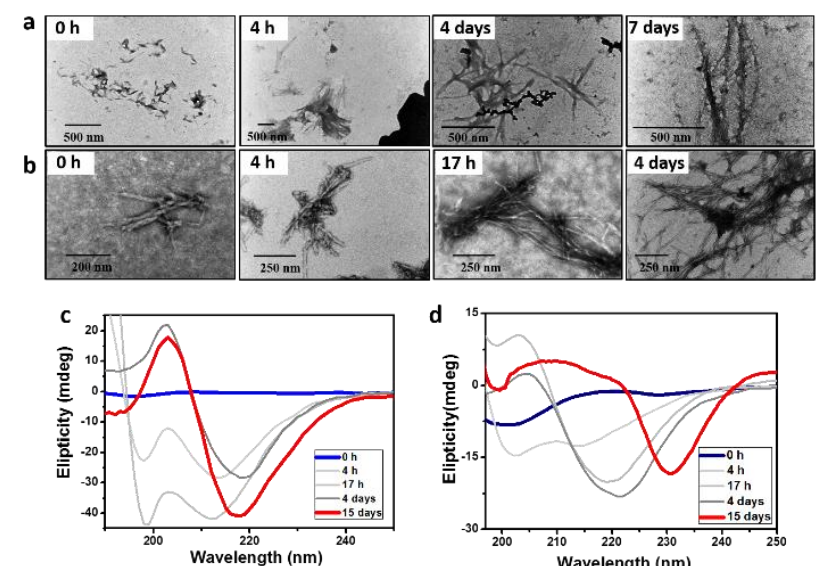

Fig. $2(a, b)$ Kinetics of fiber formation as observed by TEM for $A \beta(16$ 22) (a) and $A \beta(22-16)$ (b). (c,d) CD spectra showing the transition from unstructured conformation into $\beta$-sheet over time for $\mathrm{A} \beta(16-22)$ (c) and $\mathrm{A} \beta(22-16)(\mathrm{d})$.

pathogenesis, recent evidence suggests oligomeric intermediates are the most toxic species. ${ }^{14}$ Our kinetic study evidently showed that the rate of self-assembly and mature fiber formation of the native peptide was slow. Throughout the MTT assay, most peptide molecules were in an intermediate oligomeric state, thus leading to cell toxicity. However, the reverse peptide selfassembled at a much faster rate and most of the peptide molecules transformed into mature fibers during the experimental time scale, thus resulting in no significant cell toxicity. We also examined the stability of the two peptides in the presence of human serum. ${ }^{15}$ The results indicate both peptides to be quite stable as no degradation was observed up to $72 \mathrm{~h}$ in the presence of proteolytic enzymes (ESI Figure S5, S6). We studied the membrane interactions of the peptides to explore the mechanism of toxicity. ${ }^{1 \mathrm{~d}}$ For this, we used vesicles mimicking mammalian plasma membranes comprising 1,2dioleoyl-sn-glycero-3-phosphocholine (DOPC), cholesterol, and sphingomyelin (DOPC/Chol/Sph). Fig. 3c-f shows the fundamental differences in membrane interactions of the two peptides. Although both peptides fibrillized in either the presence or the absence of lipid bilayers, $A \beta(16-22)$ showed intimate interactions with lipid bilayers. As shown in Fig. 3c,d, the vesicles and fibrils of $A \beta(16-22)$ were bound, and no free vesicles are found. In contrast, for $A \beta(22-16)$, only a small amount of lipid vesicles adhered to the fibrillar structures (Fig. 3e,f and ESI Figure S7 ). This difference in the interactions is probably due to the slightly different surface potential originating from the assembly state and structure which induced different organization of the charged residues on the oligomer/fibril surface.

To elucidate the influence of lipid bilayer on peptide assembly, the kinetics of fibrillation was studied using Thioflavin T (ThT) fluorescence assay (Fig. 3g). Generally, ThT fluorescence increases in the presence of amyloidal fibrillar structures. ${ }^{16}$ Likewise, increase in ThT fluorescence during a kinetic measurement indicates a process of fibril elongation. ${ }^{17}$ The reverse peptide did not display any fluorescence increase during
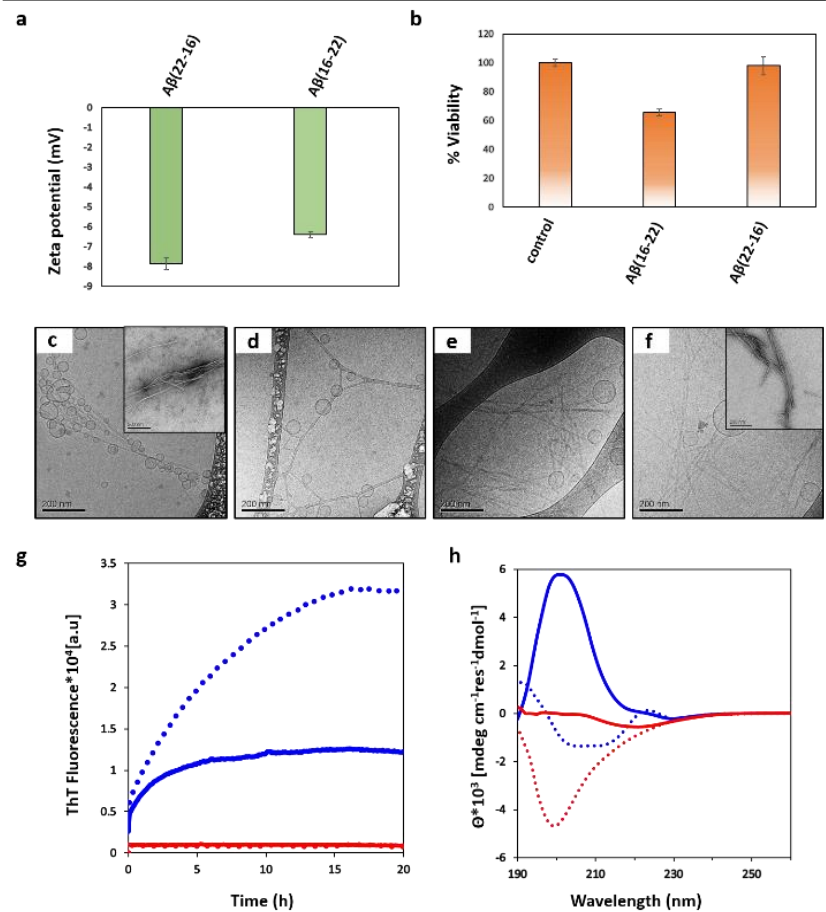

Fig. 3 (a) $\zeta$-potential of the two peptides. (b) MTT cell viability assay. (c-f) Cryo-TEM of $A \beta(16-22)(c, d)$ and $A \beta(22-16)(e, f)$ in the presence of lipid vesicles. Insets show negatively stained images of the peptides only. (g) Kinetics of peptide fibrillation using ThT fluorescence. (h) CD spectroscopy secondary structure analysis of $A \beta(16-22)$ and $A \beta(22-16)$ with and without lipid vesicles. In $\mathrm{g}$ and $\mathrm{h}$ : Blue and red spectra represent $A \beta(16-22)$ and $A \beta(22-16)$, respectively. Broken line represents peptide only and continuous line represents peptide with lipid vesicles. Scale bar for $\mathrm{c}-\mathrm{f}$ is $200 \mathrm{~nm}$.

96 hours (only the first 20 hours are shown). This may be due to fast fibrillation and sedimentation of the aggregates. Nevertheless, the particular surface potential may also induce ThT adherence to the fibril surface without flattening the ThT probe to its fluorescent conformation. Moreover, the addition of lipid vesicles did not result in any change in ThT fluorescence. In contrast, $A \beta(16-22)$ showed a dramatic increase in fluorescence (dotted blue curve). This increase in florescence intensity is related to typical amyloid fibril elongation. Surprisingly, the addition of lipid vesicles resulted in inhibition of the fibrillation process, as exemplified by the reduction of fluorescence in the presence of lipid vesicles, as compared to their absence. The lowered maximal fluorescence indicates that addition of lipid vesicles resulted in inhibition of the fibril elongation step, rather than inhibition of early stages of aggregation from monomer to oligomer.

To evaluate the impact of the lipid membranes on peptide conformation, CD spectra were recorded (Fig. 3h). The reverse peptide showed a spectrum with a minimum around $197 \mathrm{~nm}$, correlated to a random coil structure. The presence of lipid vesicles resulted in a $C D$ spectrum with minimum at $220 \mathrm{~nm}$. However, the signal was extremely weak, possibly due to very low solubility of the peptide assemblies. The significant phase separation between peptide nanostructures and lipid vesicles was also identified in cryo-TEM images. In contrast, the random coil 
conformation of the native peptide without lipid vesicles (broken blue line in Fig. 3h) changed dramatically in the presence of lipids, with the strong maximum at $\sim 205 \mathrm{~nm}$ indicating the formation of a $\beta$-sheet like structural pattern with exposed Phe sidechains. ${ }^{18}$ Thus, the formation of $\beta$-sheet structure by the native peptide is not affected by the presence of lipid vesicles, in contrast to the reverse peptide.

It has been shown that the effects of lipids on amyloid aggregation are diverse, among which modulating of aggregation pathways is the most significant. ${ }^{19}$ The charged membranes act as two-dimensional aggregation-templates to initiate the soluble random coil monomeric peptides to aggregate into $\beta$-sheet oligomers at a faster rate for native peptide. However, ThT kinetics suggests that the fibril elongation from the oligomeric state is inhibited in the presence of lipid bilayers. The heterotypic aggregation (peptide-lipid) follows an off-fibril formation pathway instead of homotypic (peptide-peptide) on-pathway. "Off-pathway" oligomers tend to have a longer half-life and are slow in converting into mature fibrils due to their potential stabilization in kinetic traps. Thus, the presence of lipid vesicles significantly alters the aggregation pathways for the native peptide and induces oligomer-stabilizing conditions, which in turn causes amyloid cytotoxicity.

In conclusion, our work sheds light on the observation that although the high toxicity of the $A \beta(1-42)$ peptide leads to neuronal dysfunction, the reverse sequence $A \beta(42-1)$ is nontoxic, which has been assumed to be due to its inability to form ordered fibers. Using a core recognition motif of $A \beta$, here we demonstrate that although both peptide sequences aggregate to form fibrillar structures, they display markedly different aggregation kinetics. Moreover, the native peptide, not the reverse, shows strong cellular cytotoxicity. A membrane binding study reveals that the native peptide shows a strong affinity to lipid vesicles, resulting in stabilization of the oligomeric state. In contrast, the reverse peptide forms mature fibers at a much faster rate and is phase separated from the lipid vesicles. It has minimal affinity for the bilayer lipid vesicles and therefore does not show any toxicity. This study provides a firm foundation for the understanding of the influence of amino acid sequence order on amyloid assembly and function, which may pave the way to indepth understanding of the amyloid formation process.

This project received funding from ERC (grant agreement No BISON-694426 to E.G). I.W.H thanks EPSRC (UK) for the award of a Platform grant (EP/L020599/1).

\section{Conflicts of interest}

There are no conflicts to declare.

\section{Notes and references}

1. (a) D. B. Teplow, Amyloid: International Journal of Experimental and Clinical Investigation, 1998, 5, 121; (b) D. J. Selkoe, Nature Medicine, 2011, 17, 1060; (c) F. Chiti and C. M. Dobson, Annu. Rev. Biochem., 2006, 75, 333; (d) I. W. Hamley, Chem. Rev. 2012, 112, 5147; (e) G. Wei, Z. Q. Su, N. P.
Reynolds, P. Arosio, I. W. Hamley, E. Gazit and R. Mezzenga, Chem. Soc. Rev., 2017, 46, 4661.

2. (a) C. Hilbich, B. Kisterswoike, J. Reed, C. L. Masters and K. Beyreuther, J. Molec. Biol. 1992, 228, 460; (b) K. Tao, J. Wang, P. Zhou, C. Wang, H. Xu, X. Zhao and J. R. Lu, Langmuir, 2011, 27, 2723.

3. (a) A. K. Mehta, K. Lu, W. S. Childers, S. Liang, J. Dong, J. P. Snyder, S. V. Pingali, P. Thiyagarajan and D. G. Lynn, J. Am. Chem. Soc., 2008, 130, 9829; (b) K. Lu, J. Jacob, P. Thiyagarajan, V. P. Conticello and D. G. Lynn, J. Am. Chem. Soc., 2003, 125, 6391.

4. (a) E. Gazit, FASEB Journal, 2002, 16, 77; (b) E. Gazit, FEBS Journal, 2005, 272, 5971.

5. (a) V. Castelletto, I. W. Hamley and P. J. F. Harris, Biophys. Chem., 2008, 138, 29; (b) I. W. Hamley, D. R. Nutt, G. D. Brown, J. F. Miravet, B. Escuder and F. Rodríguez-Llansola, $J$. Phys. Chem. B, 2010, 114, 940.

6. (a) S. M. Butterfield and H. A. Lashuel, Angew. Chem., 2010, 49, 5628; (b) K. Matsuzaki, Biochim. Biophys. Acta-Biomembr., 2007, 1768, 1935.

7. (a) D. M. Vadukul, O. Gbajumo, K. E. Marshall and L. C. Serpell, FEBS Lett., 2017, 591, 822; (b) M. Emre, C. Geula, B. J. Ransil and M. M. Mesulam, Neurobiol. Aging, 1992, 13, 553; (c) C. Ripoli, S. Cocco, D. D. Li Puma, R. Piacentini, A. Mastrodonato, F. Scala, D. Puzzo, M. D'Ascenzo and C. Grassi, J. Neurosci., 2014, 34, 12893-12903.

8. (a) S. R. Paik, J. H. Lee, D. H. Kim, C. S. Chang and Y. S. Kim, FEBS Lett., 1998, 421, 73; (b) D. H. Bergin, Y. Jing, H. Zhang and P. Liu, Neuroscience, 2015, 298, 367.

9. M. Taylor, S. Moore, J. Mayes, E. Parkin, M. Beeg, M. Canovi, M. Gobbi, D. M. A. Mann and D. Allsop, Biochemistry, 2010, 49, 3261.

10. L. L. Yu, Y. F. Zheng, J. Xu, F. Y. Qu, Y. C. Lin, Y. M. Zou, Y. L. Yang, S. L. Gras and C. Wang, Nano Res., 2018, 11, 577. 11. V. Castelletto, I. W. Hamley, In Peptide Self-Assembly: Methods and Protocols, B. L. Nilsson, T. M. Doran, Eds. Humana Press Inc: Totowa, 2018; Vol. 1777, pp 3-21.

12. M.-C. Hsieh, C. Liang, A. K. Mehta, D. G. Lynn and M. A. Grover, J. Am. Chem. Soc., 2017, 139, 17007.

13. (a) L. K. Simmons, P. C. May, K. J. Tomaselli, R. E. Rydel, K. S. Fuson, E. F. Brigham, S. Wright, I. Lieberburg, G. W. Becker, D. N. Brems and W. Y. Li, Mol. Pharmacol., 1993, 45, 373; (b) C. Soto and E. M. Castaño, Biochem. J., 1996, 314, 701. 14. (a) M. Bucciantini, E. Giannoni, F. Chiti, F. Baroni, L. Formigli, J. Zurdo, N. Taddei, G. Ramponi, C. M. Dobson and M. Stefani, Nature, 2002, 416, 507; (b) C. M. Dobson, Trends Biochem. Sci. 1999, 24, 329.

15. A. Paul, S. Kalita, S. Kalita, P. Sukumar and B. Mandal, Sci. Rep., 2017, 7, 40095.

16. H. III LeVine, Protein Sci., 1993, 2, 404.

17. T. P. J. Knowles, C. A. Waudby, G. L. Devlin, S. I. A. Cohen, A. Aguzzi, M. Vendruscolo, E. M. Terentjev, M. E. Welland and C. M. Dobson, Science, 2009, 326, 1533.

18. M. J. Krysmann, V. Castelletto and I. W. Hamley, Soft Matter, 2007, 3, 1401.

19. V. Rangachari, D. N. Dean, P. Rana, A. Vaidya and P. Ghosh, Biochim. Biophys. Acta Biomembr., 2018, 1860, 1652. 